\title{
Puerarin prevents cataract development and progression in diabetic rats through $\mathrm{Nrf2/HO-1}$ signaling
}

\author{
DUZHEN ZHANG and MAN LI \\ Department of Ophthalmology, Linyi Central Hospital, Linyi, Shandong 276400, P.R. China
}

Received November 3, 2018; Accepted April 17, 2019

DOI: $10.3892 / \mathrm{mmr} .2019 .10320$

\begin{abstract}
Puerarin is the major bioactive ingredient isolated from the dry root of Pueraria lobata, a plant used in traditional Chinese medicine. Puerarin has been used to treat diabetes and cataracts in China; however, its underlying mechanism of action remains unclear. The aim of the present study was to investigate the effectiveness and mechanism of puerarin in preventing cataracts in diabetic rats. Diabetes was induced by streptozocin (STZ) administration and rats were intraperitoneally injected with puerarin $(25,50$ and $100 \mathrm{mg} / \mathrm{kg})$. Blood glucose levels and cataract development were examined in the different experimental groups. In addition, the expression levels of markers associated with oxidative stress, including nuclear factor erythroid 2 like 2 (Nrf2) and heme oxygenase-1 (HO-1), were analyzed. The present results suggested that treatment with puerarin at 25,50 and $100 \mathrm{mg} / \mathrm{kg}$ significantly reduced blood glucose levels and the incidence of cataract in STZ-induced diabetic rats. Additionally, puerarin treatment reduced oxidative stress, restoring the levels of malondialdehyde and glutathione, and the activity of glutathione peroxidase. Furthermore, puerarin administration decreased the expression levels of retinal vascular endothelial growth factor and interleukin- $1 \beta$ and increased the mRNA expression levels of Nrf2 and HO-1, thus inhibiting oxidative stress. The present findings suggested that puerarin had hypoglycemic effects and that it prevented cataract development and progression in diabetic rats by reducing oxidative stress through the Nrf2/HO-1 signaling pathway.
\end{abstract}

\section{Introduction}

Hyperglycemia is an important factor involved in the development of cardiovascular disease, kidney failure and cataract (1). Patients with diabetes are more likely to develop cataracts

Correspondence to: Professor Duzhen Zhang, Department of Ophthalmology, Linyi Central Hospital, 17 Jian Kang Road, Linyi, Shandong 276400, P.R. China

E-mail: zhang_dz66@sina.com

Key words: diabetes, cataract, puerarin, oxidative stress, nuclear factor erythroid 2 like 2 /heme oxygenase-1 signaling compared with healthy subjects (2). Diabetic retinopathy is characterized by a dysfunctional local microcirculation, which causes structural changes in the microvasculature, leading to the morphological alterations of large vessels, including arteries and veins (3). Furthermore, morphological changes can induce vascular lesions in the retina (3). Therefore, since the number of diabetic patients is increasing worldwide, the occurrence rate of diabetic cataract is also increasing (4).

Multiple factors contribute to cataract development, including aging, smoking (5), exposure to ultraviolet-B and ionizing radiations and oxidative stress (6). Diabetic cataracts are induced by a glutathione (GSH) deficiency that impairs the antioxidative mechanisms in the lens of the eye (7). Additionally, deficiency of nutrients, including vitamins E (8), A (9) and C (10), and selenium, can also lead to cataract development.

Puerarin is an isoflavone found at high concentrations in the dry root extract of Pueraria lobata (Willd) Ohwi, and was first described in the 1950s as the main active ingredient in Radix Pueraria lobata (11). Puerarin is commonly used in the treatment of cerebrovascular disorders, cardiovascular disease (12), cancer (13), diabetes and diabetes-associated complications (14). Puerarin eyedrops have been used to treat eye diseases in China for years (15). Several previous studies have demonstrated that puerarin protects against oxidative damage, inflammation and hyperlipidemia $(16,17)$. The beneficial effects of puerarin may be due to its ability to decrease blood glucose levels, reduce insulin resistance and scavenge oxygen free radicals in diabetic rats $(18,19)$.

Puerarin toxicity is limited, and this safe natural compound has been shown to inhibit diabetic ocular complications, such as cataract $(20,21)$. Therefore, over the past decade, an increasing number of studies have investigated the pharmacological effects of puerarin (22). A previous study demonstrated that puerarin ameliorates streptozocin (STZ)-induced diabetic retinopathy in rats by decreasing the mRNA or protein expression levels of various factors in the rat retina, including Fas cell surface death receptor, Fas ligand, nitrotyrosine, inducible nitric oxide synthase (23), advanced glycation end product receptors and vascular endothelial growth factor (VEGF) $(19,20)$. Additionally, puerarin decreases the apoptotic rate of retinal pigment epithelial cells in diabetic rats induced by STZ injection $(24,25)$. The aim of the present study was to investigate the pharmacological mechanism underlying puerarin function in inhibiting cataract development. In particular, the 
present study examined the nuclear factor erythroid 2 like 2 (Nrf2)/heme oxygenase (HO-1) signaling pathway.

\section{Materials and methods}

Animal experiment. A total of 90 Male Wistar rats (6-8 weeks, 180-220 g) were purchased from The Shanghai Animal Center, and kept under specific pathogen-free conditions. The rats were housed at $22 \pm 2^{\circ} \mathrm{C}$, under a 12 -h light/dark cycle, at $50 \pm 10 \%$ relative humidity, and with free access to water and food. Experiments were performed according to the guidelines for the care and use of experimental animals established by the Ministry of Science and Technology of the People's Republic of China (26), and the study was approved by the Laboratory Animal Management Committee of Linyi Central Hospital (approval no. 2006-398). After 2 weeks of acclimatization, animals were administered $65 \mathrm{mg} / \mathrm{kg}$ STZ (Sigma-Aldrich; Merck KGaA) in $0.1 \mathrm{M}$ citrate buffer ( $\mathrm{pH} 4.5$ ), as previously described (27-29). Rats in the control group were administered $0.1 \mathrm{M}$ citrate buffer (vehicle control). Blood glucose was measured every week using a GlucoLeader automatic analyzer. In the present study, animals exhibiting blood glucose levels $\geq 16 \mathrm{mmol} / 1$ were considered diabetic (30).

Subsequently, animals treated with STZ were randomly divided into four experimental groups. Diabetic rats (18 rats in each group) were administered with 0 (DM group; untreated diabetic rats), 25, 50 or $100 \mathrm{mg} / \mathrm{kg}$ puerarin (Aladdin Reagent) by intraperitoneal injection, as previously described $(31,32)$. Animals were treated with puerarin for 12 weeks daily, and they were provided a standard rodent diet with free access to water. Untreated non-diabetic rats were also used as the control group with an equal volume of $0.1 \mathrm{M}$ citrate buffer. Blood was collected from the tail vein, and blood glucose levels were monitored in all rats.

Evaluation of cataract development. After 12 weeks, the development of cataract was assessed using an ophthalmoscope. Eye inspection was preceded by topical administration of $1 \%$ tropicamide drops. Cataract formation was graded based on the classification described by Varma (33): Grade 0 , clear normal lens; grade I, peripheral vesicles; grade II, peripheral vesicles and cortical opacities; grade III, diffuse central opacities; and grade IV, mature cataract. Cataract formation was considered complete (grade IV) when the red fundus reflex was not visible through any part of the lens and the lens appeared completely opaque to the naked eye.

Lens preparation. After 12 weeks, the rats were sacrificed and the eye lenses were collected. Each pair of lenses was homogenized in prechilled $0.2 \mathrm{M}$ potassium phosphate buffer ( $\mathrm{pH}$ 7.0). This homogenate was used to assess the activity of glutathione peroxidase (GPx), the concentrations of GSH and malondialdehyde (MDA), and the protein expression levels of various factors. Samples were stored at $-80^{\circ} \mathrm{C}$ prior to use in biochemical assays.

Measurement of oxidative stress markers. Antioxidant capacity (AOC) was measured using total Antioxidant capacity assay kit (A015-1-2, Nanjing Jiancheng Bioengineering Institute) according to the manufacturer's protocol. The assay is based on the ability of the sample to inhibit oxidation of 2,2'-azino-di(3-ethylbenzthiazoline-6-sulphonate) (ABTS) by metmyoglobin. The antioxidants in the sample cause a decrease in absorbance at $750 \mathrm{~nm}$, corresponding to the concentration of ABTS (34). Each sample was measured in duplicate.

MDA levels were measured using a thiobarbituric acid (TBA) assay kit (A003-1-2, Nanjing Jiancheng Bioengineering Institute) according to the manufacturer's protocol. Briefly, $0.1 \mathrm{ml}$ sample was mixed with 1,1,3,3-tetramethoxypropane, $0.75 \mathrm{ml}$ TBA working solution (0.37\%) and perchloric acid. The resulting solution was incubated at $95^{\circ} \mathrm{C}$ for $45 \mathrm{~min}$. After cooling (10 $\mathrm{min}$ in ice water bath), the flocculent precipitate was removed by centrifugation $(4,000 \mathrm{x} \mathrm{g} 10 \mathrm{~min}$ at room temperature). The supernatant was analyzed at $532 \mathrm{~nm}$ by multi-scan spectrum microplate spectrophotometer at room temperature.

GPx activity was assayed using an hydrogen peroxide assay (A005-1-2, Nanjing Jiancheng Bioengineering Institute), as previously described (35). The decrease in NADPH was assessed spectrophotometrically at $340 \mathrm{~nm}$. The reaction mixture consisted of $240 \mathrm{mU} / \mathrm{ml}$ glutathione disulfide reductase, $1 \mathrm{mM}$ GSH, $0.15 \mathrm{mM}$ NADPH in $0.1 \mathrm{M}$ potassium phosphate buffer ( $\mathrm{pH}$ 7.0) containing $1 \mathrm{mM}$ EDTA. In total, $50 \mu \mathrm{l}$ sample was added to this mixture and incubated at $37^{\circ} \mathrm{C}$ for $3 \mathrm{~min}$. Subsequently, $1.5 \mathrm{mM}$ hydrogen peroxide was added to adjust the final volume of the assay mixture to $1 \mathrm{ml}$.

GSH content of lens homogenate was assayed by spectrophotometric method, using the reduced GSH assay kit (A006-1-1, Nanjing Jiancheng Bioengineering Institute) according to the manufacturer's protocol. The detection was performed at $420 \mathrm{~nm}$ by multi-scan spectrum microplate spectrophotometer at room temperature.

Inflammation biochemical assays. Interleukin (IL)-1 $1 \beta$ levels were quantified in the retina using a rat IL-1 $\beta$ ELISA kit (H002, Nanjing Jiancheng Bioengineering Institute, Nanjing, China) according to the manufacturer's protocol. The concentration of IL- $1 \beta$ was calculated as pg IL- $1 \beta / \mathrm{mg}$ total retina protein in each sample according to the manufacturer's protocol. VEGF levels were quantified in the retina using a rat VEGF ELISA kit (H044, Nanjing Jiancheng Bioengineering Institute) according to the manufacturer's protocol. The standard solution or the samples were added in a 96-well plate that was coated with a monoclonal antibody from the kit. The samples were incubated for $2 \mathrm{~h} 37^{\circ} \mathrm{C}$. After washing, the samples were detected at the wavelength of $450 \mathrm{~nm}$ using a VERSA max tunable microplate reader (Molecular Devices, LLC). The ELISA assay could detect concentrations of VEGF $\geq 15 \mathrm{ng} / \mathrm{ml}$.

Reverse transcription-quantitative $P C R$ (RT-qPCR) analysis. The mRNA expression levels of Nrf2 and HO-1 were determined by RT-qPCR. Total RNA was extracted from rat retinas using TRIzol reagent (Invitrogen; Thermo Fisher Scientific, Inc.). RT was performed using $1 \mu \mathrm{g}$ RNA. Moloney Murine Leukemia Virus reverse transcriptase kit (cat. no. 28025013, Thermo Fisher Scientific, Inc.) was used to synthesize the cDNA. The synthesized cDNA was diluted by adding $75 \mu \mathrm{l}$ DNase-free water and stored at $-20^{\circ} \mathrm{C}$. qPCR was performed using the SYBR-Green PCR kit (Takara Biotechnology Co., Ltd.) and gene-specific primers were 
synthesized by Nanjing Sunshine Biotechnology Co., Ltd. Primer sequences are listed in Table I. The thermocycling conditions were: $95^{\circ} \mathrm{C}$ for $10 \mathrm{~min}, 95^{\circ} \mathrm{C}$ for $10 \mathrm{sec}$ and $55^{\circ} \mathrm{C}$ for $10 \mathrm{sec}$, with a final step of $72^{\circ} \mathrm{C}$ for $30 \mathrm{sec}$. Then steps $2-4$ were repeated for 40 cycles followed by a melt curve program for $60 \mathrm{~min}$. GAPDH served as an internal control. Relative gene expression levels of Nrf2 and HO-1 were measured using the $2^{-\Delta \Delta \mathrm{Cq}}$ method (36). The expression levels of Nrf2 and HO-1 were normalized to the control group.

Western blot analysis. For immunoblot analysis, total protein was extracted using radioimmunoprecipitation buffer (P0013D, Beyotime Institute of Biotechnology) and nuclear extracts were isolated with a Nuclear and Cytoplasmic Protein Extraction kit (BioTeke Corporation), according to the manufacturer's instructions. Equal amounts of protein samples $(\sim 30 \mu \mathrm{g})$ were separated by $10 \%$ SDS-PAGE. The protein concentration was determined using a bicinchoninic protein assay according to the manufacturer's protocol (Thermo Fisher Scientific, Inc.). The proteins were electro-blotted onto a PVDF membrane (thickness, $0.45 \mu \mathrm{m}$; EMD Millipore). The membranes were incubated with blocking solution (5\% dried milk) for $1 \mathrm{~h}$ at room temperature and incubated overnight at $4^{\circ} \mathrm{C}$ with specific primary antibodies anti-Nrf2 $(1: 1,000$; cat. no. ab137550; Abcam), HO-1 (1:1,000; cat. no. ab13248; Abcam), GAPDH (1:5,000, cat. no. A0208; Beyotime Institute of Biotechnology) and LaminB (1:5,000, cat. no. AF1408; Beyotime Institute of Biotechnology). After washing with TBS $/ 0.05 \%$ Tween 20 three times, the blots were incubated with horseradish peroxidase-conjugated immunoglobulin $\mathrm{G}$, horseradish peroxidase-labeled mouse anti-rabbit IgG (1:5,000; cat. no. 5127, Cell Signaling Technology, Inc.) and horseradish peroxidase-labeled rabbit anti-mouse IgG (1:5,000; cat. no. 58802, Cell Signaling Technology, Inc.), for $1 \mathrm{~h}$ at room temperature. The signals were developed using the Pierce ECL western blotting substrate (Thermo Fisher Scientific, Inc.). Immunoreactive protein bands were visualized using the ChemiDoc MP Imaging system and quantified using Image Lab 3.0 software (Bio-Rad Laboratories, Inc.).

Statistical analysis. Data are presented as the mean \pm standard error of the mean. Comparisons were performed using one-way ANOVA followed by Tukey's post hoc test using GraphPad Prism software 6.0 (GraphPad Software, Inc.). $\mathrm{P}<0.05$ was considered to indicate a statistically significant difference.

\section{Results}

Blood glucose measurement. Blood glucose levels in all experimental animal groups were measured every week. Puerarin administration significantly decreased blood glucose levels in diabetic rats, between 6 and 12 weeks of STZ administration (Fig. 1).

Cataract development and progression. The effects of puerarin on cataract development in hyperglycemic rats were examined (Fig. 2). First, the incidence of cataract in diabetic rats following treatment with puerarin was investigated (Table II). Each group consisted of 18 rats. A total of 15 rats developed
Table I. Gene-specific PCR primer sequences.

\begin{tabular}{ll}
\hline Gene symbol & \multicolumn{1}{c}{ Sequence (5'-3') } \\
\hline Nrf2 & F: TTCCTCTGCTGCCATTAGTCAGTC \\
& R:GCTCTTCCATTTCCGAGTCACTG \\
HO-1 & F: CTGGAAGAGGAGATAGAGCGAA \\
& R:TCTTAGCCTCTTCTGTCACCCT \\
GAPDH & F: TGATGACATCAAGAAGGTGGTGA \\
& R:TCCTTGGAGGCCATGTAGGCCAT \\
LMNB1 & F: CCGGATGGTGGGGCTTTGTT \\
& R:CCGGGCCTCTCGATGGATAAGC
\end{tabular}

F, forward; R, reverse; Nrf2, nuclear factor erythroid 2 like 2; HO-1, heme oxygenase-1; LMNB, lamin B.

Table II. Effect of puerarin on the incidence of cataract in streptozocin-induced diabetic rats.

\begin{tabular}{lc} 
Groups & $\begin{array}{c}\text { Cataract incidence (number } \\
\text { of animals with cataract) }\end{array}$ \\
\hline Diabetic & $15 / 18$ \\
Diabetic $+25 \mathrm{mg} / \mathrm{kg}$ puerarin & $13 / 18$ \\
Diabetic $+50 \mathrm{mg} / \mathrm{kg}$ puerarin & $11 / 18$ \\
Diabetic $+100 \mathrm{mg} / \mathrm{kg}$ puerarin & $7 / 18$
\end{tabular}

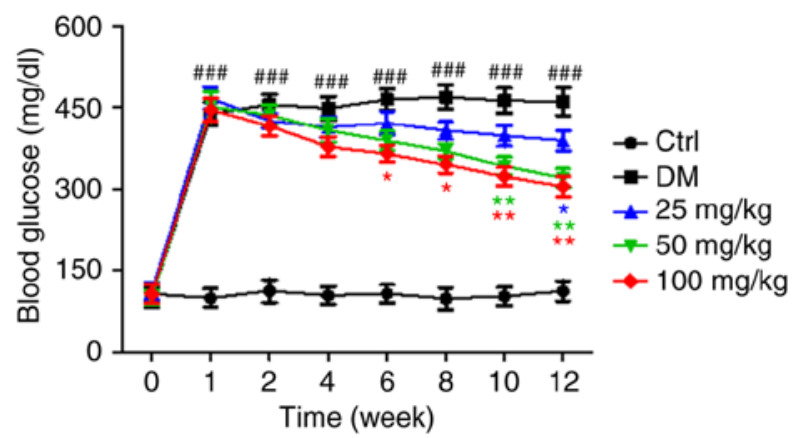

Figure 1. Effect of puerarin on blood glucose levels in the different experimental groups. Data are presented as the mean \pm standard error of the mean ( $\mathrm{n}=18$ rats/group). ${ }^{\# \# \#} \mathrm{P}<0.001$ vs. $\mathrm{Ctrl} ;{ }^{*} \mathrm{P}<0.05,{ }^{* *} \mathrm{P}<0.01$ vs. $\mathrm{DM}$. $\mathrm{Ctrl}$, untreated control rats; DM, untreated diabetic rats.

cataract in the diabetic group (rats induced withSTZ and not treated with puerarin). Following treatment with puerarin at 25, 50 and $100 \mathrm{mg} / \mathrm{kg}$, the number of rats with cataracts was 13,11 and 7, respectively (Table II). All animals presented clear lenses (baseline cataract score, 0 ) at week 0 . However, after 12 weeks, the cataract scores varied among groups (Table III). The average cataract scores at the end of the experiment were decreased in the diabetic rats treated with puerarin compared with the untreated group, particularly in the $100 \mathrm{mg} / \mathrm{kg}$ group $(\mathrm{P}<0.01)$.

Effects of puerarin on the levels of MDA, GSH, AOC and $G P x$ in the lens of diabetic rats. The untreated diabetic group 
Table III. Effect of puerarin on the cataract score in streptozocin-induced diabetic rats.

\begin{tabular}{lcccc}
\hline $\begin{array}{l}\text { Cataract } \\
\text { score }\end{array}$ & Diabetic & $\begin{array}{c}\text { Diabetic }+25 \mathrm{mg} / \mathrm{kg} \\
\text { puerarin }\end{array}$ & $\begin{array}{c}\text { Diabetic }+50 \mathrm{mg} / \mathrm{kg} \\
\text { puerarin }\end{array}$ & $\begin{array}{c}\text { Diabetic }+100 \mathrm{mg} / \mathrm{kg} \\
\text { puerarin }\end{array}$ \\
\hline 0 & 3 & 5 & 7 & 10 \\
I & 1 & 1 & 2 & 3 \\
II & 3 & 4 & 4 & 2 \\
III & 5 & 4 & 4 & 3 \\
IV & 6 & 4 & 1 & 0 \\
\hline
\end{tabular}
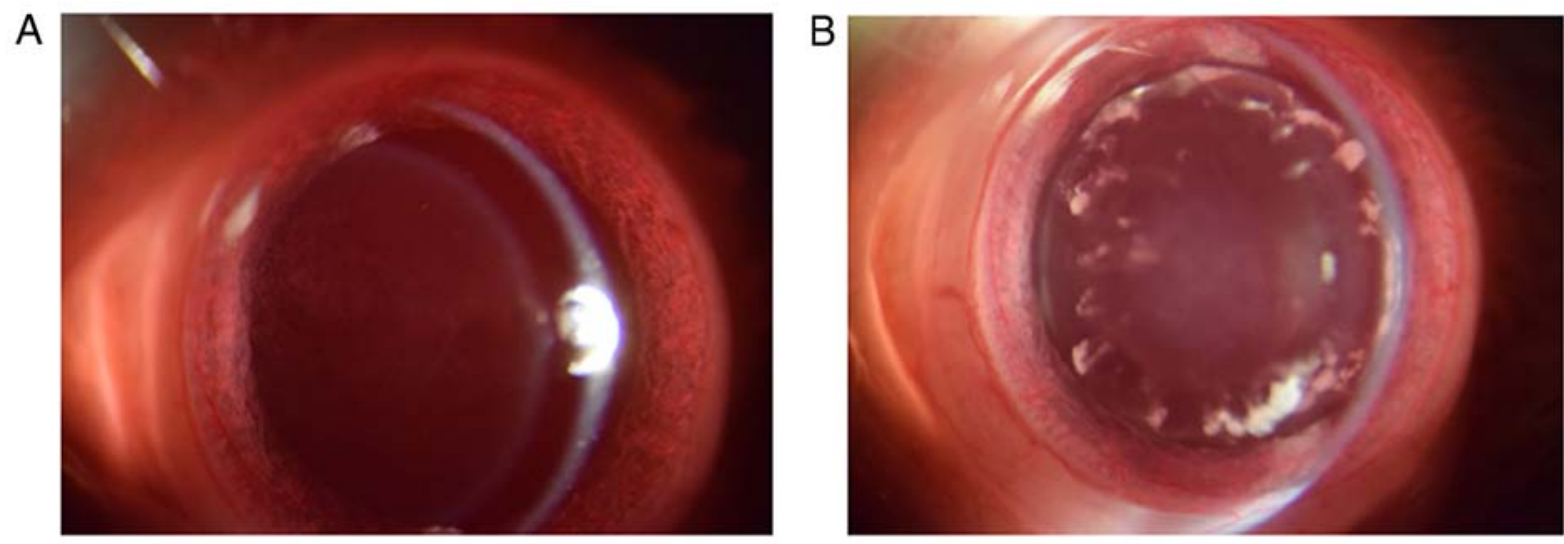

Figure 2. Photographs of lenses from a (A) normal and a (B) diabetic rat.

exhibited significantly different levels of AOC, GSH, GPx and MDA compared with the control group (Fig. 3). Moreover, in the groups treated with 50 and $100 \mathrm{mg} / \mathrm{kg}$ puerarin, AOC and GSH levels were significantly higher compared with the untreated diabetic group (Fig. 3A and B). The levels of GPx in groups treated with 50 and $100 \mathrm{mg} / \mathrm{kg}$ puerarin were significantly higher compared with the untreated diabetic group. (Fig. 3C). Finally, MDA levels were significantly decreased by treatment with 50 and $100 \mathrm{mg} / \mathrm{kg}$ puerarin, compared with the untreated diabetic group (Fig. 3D).

ELISA results of IL-1 $\beta$ and VEGF levels. To assess the protein levels of IL-1 $\beta$ and VEGF in the retina, retinas were collected from the different experimental groups at the end of the experiment and ELISA was performed. The untreated diabetic group exhibited significantly higher levels of VEGF and IL-1 $\beta$ levels compared with the control group (Fig. 4). Notably, the protein levels of IL-1 $\beta$ were significantly decreased in the 50 or $100 \mathrm{mg} / \mathrm{kg}$ puerarin group compared with the untreated diabetic group (Fig. 4B). Similarly, treatment with puerarin at 50 and $100 \mathrm{mg} / \mathrm{kg}$ decreased the VEGF levels compared with the untreated diabetic group (Fig. 4A).

Puerarin upregulates the activity of the Nrf2/HO-1 signaling pathway in the retinas of diabetic rats. The effects of puerarin on the mRNA expression levels of Nrf2 and HO-1 were determined in the retinas in the different groups (Fig. 5). The mRNA expression levels of Nrf2 and HO-1 were significantly increased in the diabetic rats compared with the control group. In addition, puerarin treatment upregulated the mRNA expression levels of Nrf2 and HO-1; however, the higher doses of puerarin (50 or $100 \mathrm{mg} / \mathrm{kg}$ ) significantly increased the mRNA expression levels of Nrf2. Treatment with puerarin at 50 and $100 \mathrm{mg} / \mathrm{kg}$ upregulated the mRNA expression levels of $\mathrm{HO}-1$ in diabetic rats.

Additionally, the protein expression levels of $\mathrm{Nrf} 2$ in the nuclei and the protein expression levels of HO-1 were examined by western blotting. The protein expression levels of Nrf2 and HO-1 were higher in the untreated diabetic group compared with the control group, but not statistically significant (Fig. 6). Treatment with puerarin significantly increased the protein expression levels of these factors. In conclusion, treatment with puerarin could activate the $\mathrm{Nrf} 2 / \mathrm{HO}-1$ signaling pathway in retinal tissues from STZ-induced diabetic rats.

\section{Discussion}

Puerarin has been used as a traditional Chinese medicine to treat diabetes mellitus (37). Many previous studies have reported the preventative effects of puerarin on cataract development $(38,39)$; however, the mechanism underlying the effects of puerarin on cataract development and progression remains unclear. In the present study, puerarin was identified to prevent cataract development and progression through the Nrf2/HO-1 signaling pathway in diabetic rats. Puerarin also decreased the blood glucose levels in STZ-induced diabetic rats. The present findings are consistent with previous studies showing that puerarin treatment exhibits hypoglycemic and retinal protective effects in diabetic rats $(40,41)$. 
A
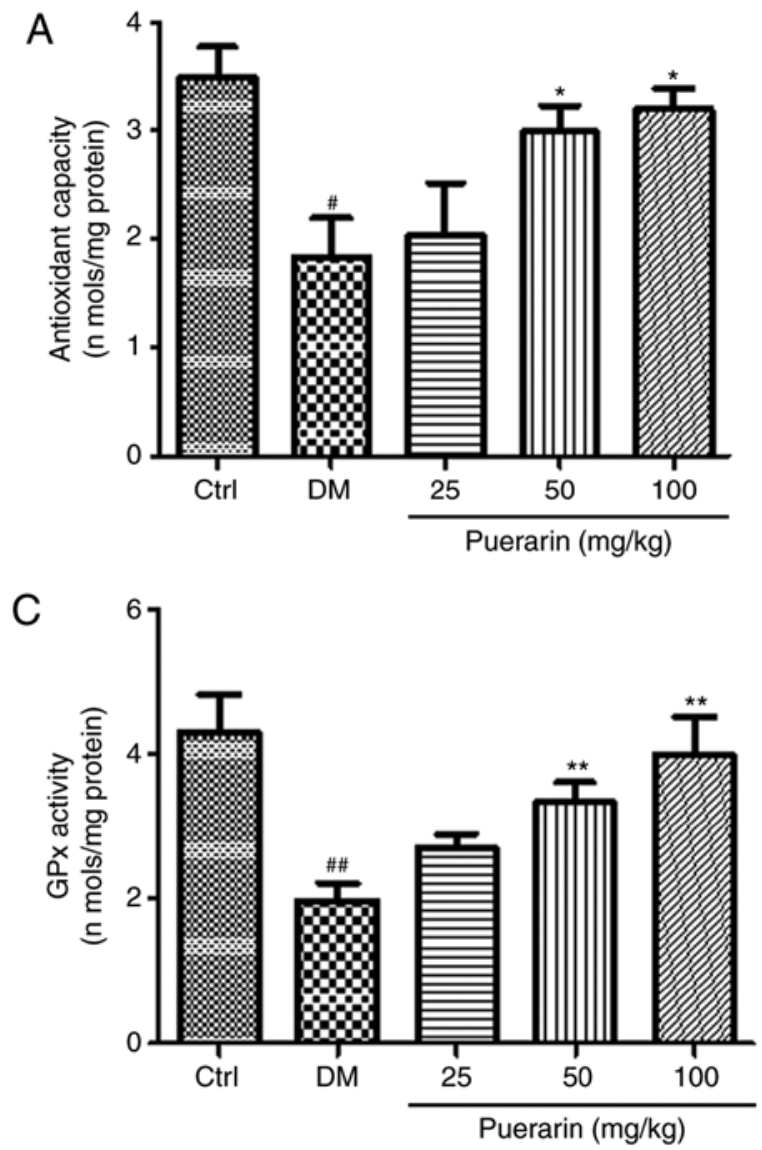

B
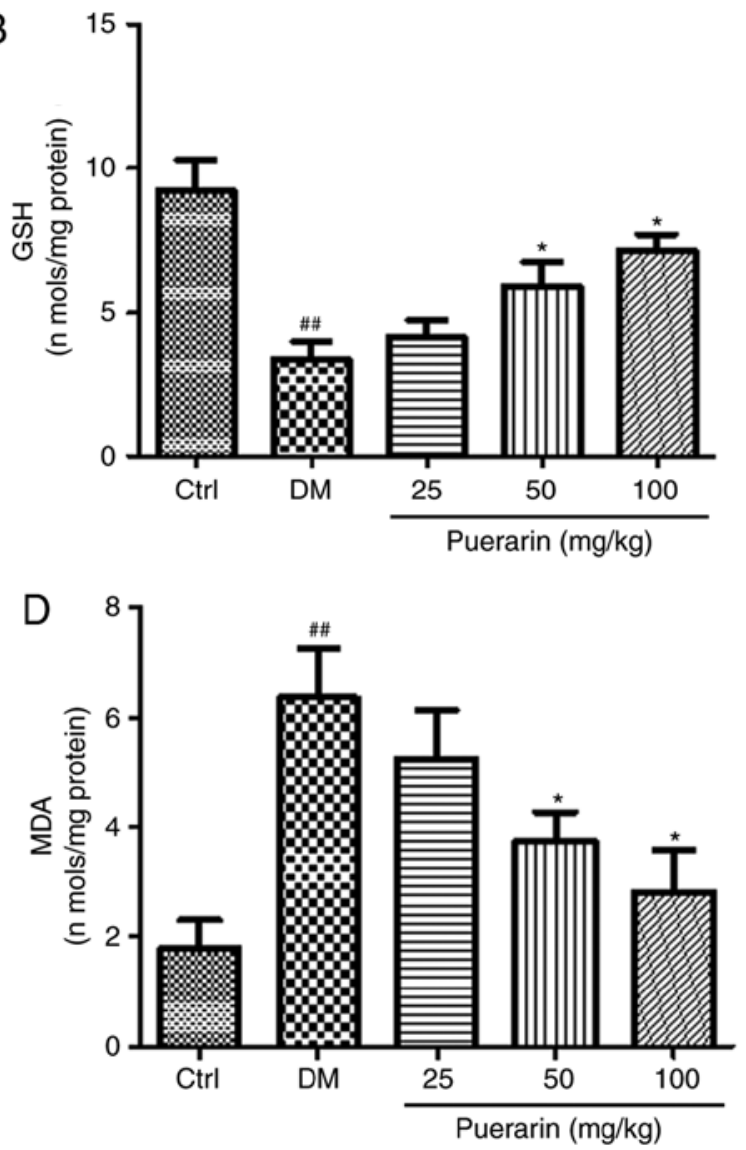

Figure 3. Effects of puerarin on diabetes-induced oxidative stress in the lens. (A) Antioxidant capacity, (B) GSH levels, (C) GPx activity and (D) MDA levels were detected using specific kits, analyzing protein homogenates of lenses collected from the different experimental groups ( $\mathrm{n}=18$ rats/group). ${ }^{*} \mathrm{P}<0.05$, ${ }^{\# \#} \mathrm{P}<0.01$ vs. Ctrl; ${ }^{*} \mathrm{P}<0.05,{ }^{* *} \mathrm{P}<0.01$ vs. DM. Ctrl, untreated control rats; DM, untreated diabetic rats; GPx, glutathione peroxidase; GSH, glutathione; MDA, malondialdehyde.
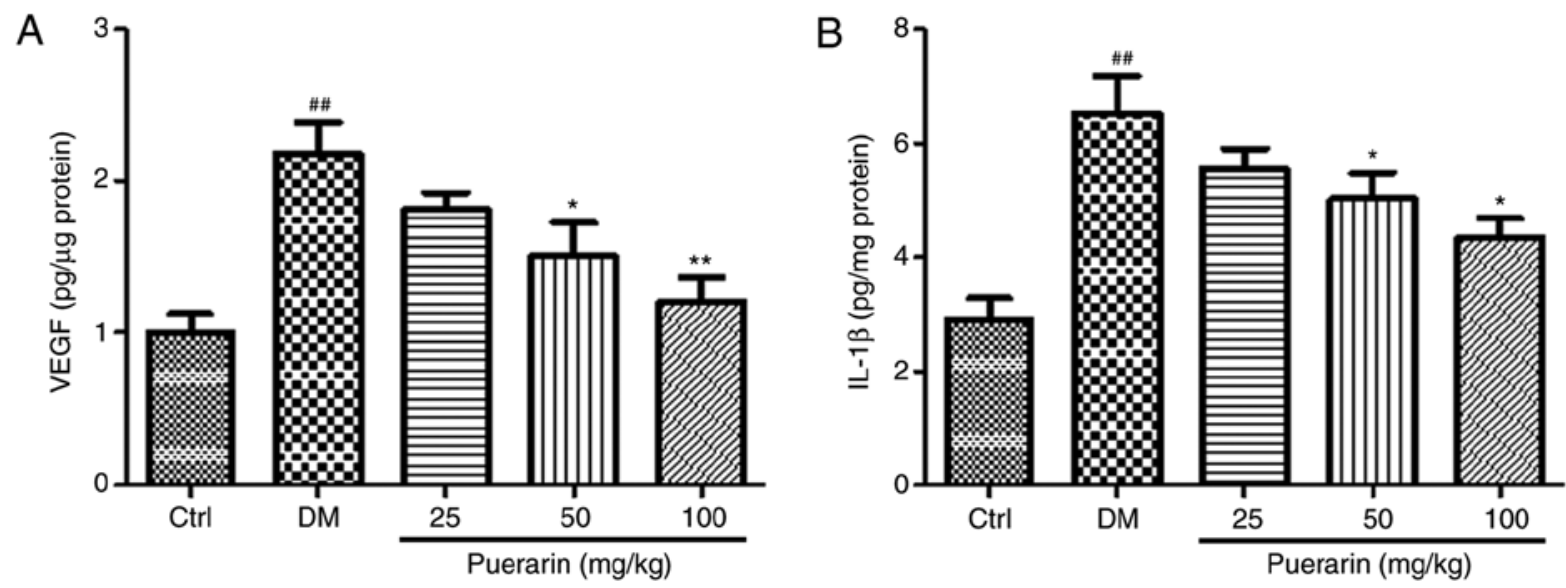

Figure 4. Protein levels of VEGF and IL-1 $\beta$. (A) VEGF and (B) IL-1 $\beta$ protein levels were measured in the retina of rats in the different experimental groups using ELISA ( $\mathrm{n}=8$ rats/group). ${ }^{\# \#} \mathrm{P}<0.01$ vs. Ctrl; ${ }^{*} \mathrm{P}<0.05,{ }^{* *} \mathrm{P}<0.01$ vs. DM. Ctrl, untreated control rats; DM, untreated diabetic rats; VEGF, vascular endothelial growth factor; IL, interleukin.

It is widely accepted that oxidative stress and free radicals are significant factors in cataract development. Specifically, oxidative stress induces protein and lipid peroxidation (42). Peroxidized lipids and proteins can form insoluble aggregates in the lens, causing the development of cataract. Lipid peroxidation has also been associated to cataract development via perturbation of the cytoplasm and cell membrane (43).
Puerarin is an isoflavone that exhibits a wide range of biological activities, including antioxidant activity (44). MDA is a marker of oxidative stress (45). In the present study, the levels of MDA in the groups treated with puerarin were decreased compared with the untreated diabetic group. Additionally, the groups treated with puerarin exhibited significantly higher AOC compared with the untreated diabetic group. GSH is 

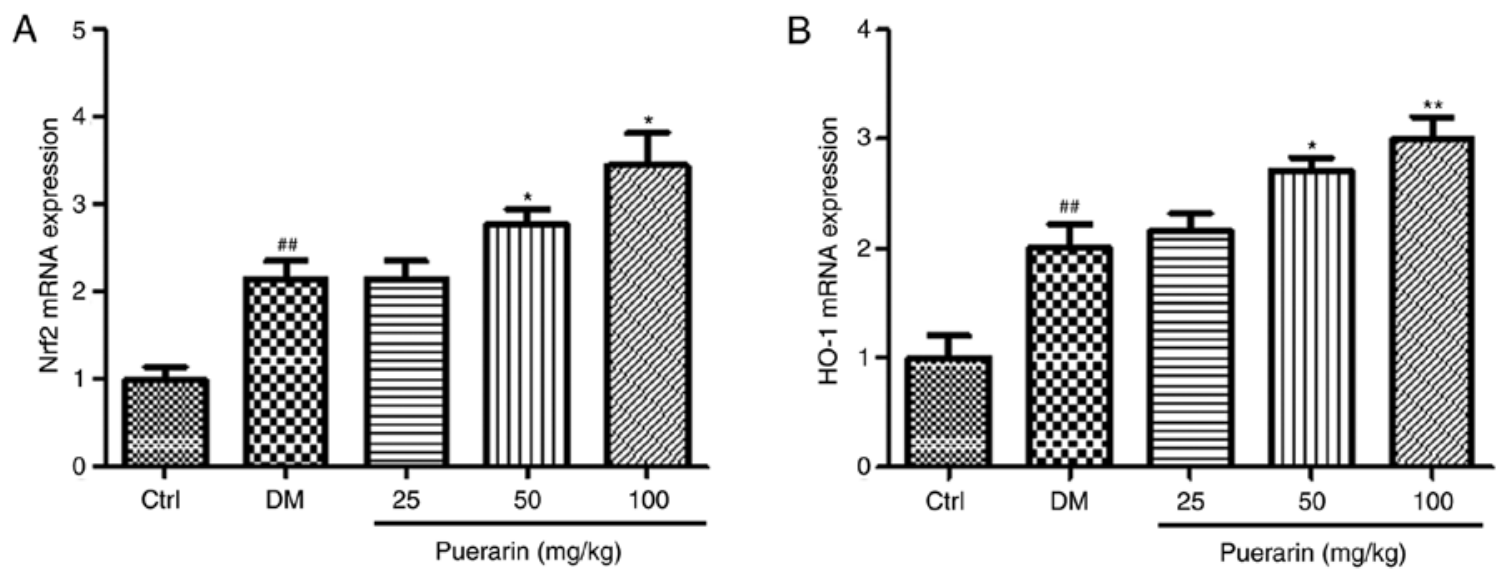

Figure 5. Puerarin administration increases the mRNA expression levels of members of the Nrf2/HO-1 signaling pathway in streptozocin-induced diabetic rats. (A) Nrf2 and (B) HO-1 mRNA expression levels were examined in retina tissues from the different experimental groups ( $\mathrm{n}=6 \mathrm{rats} / \mathrm{group}) .{ }^{\# \#} \mathrm{P}<0.01 \mathrm{vs}$. Ctrl; ${ }^{*} \mathrm{P}<0.05,{ }^{* *} \mathrm{P}<0.01$ vs. DM. Ctrl, untreated control rats; DM, untreated diabetic rats; Nrf2, nuclear factor erythroid 2 like 2; HO-1, heme oxygenase-1.
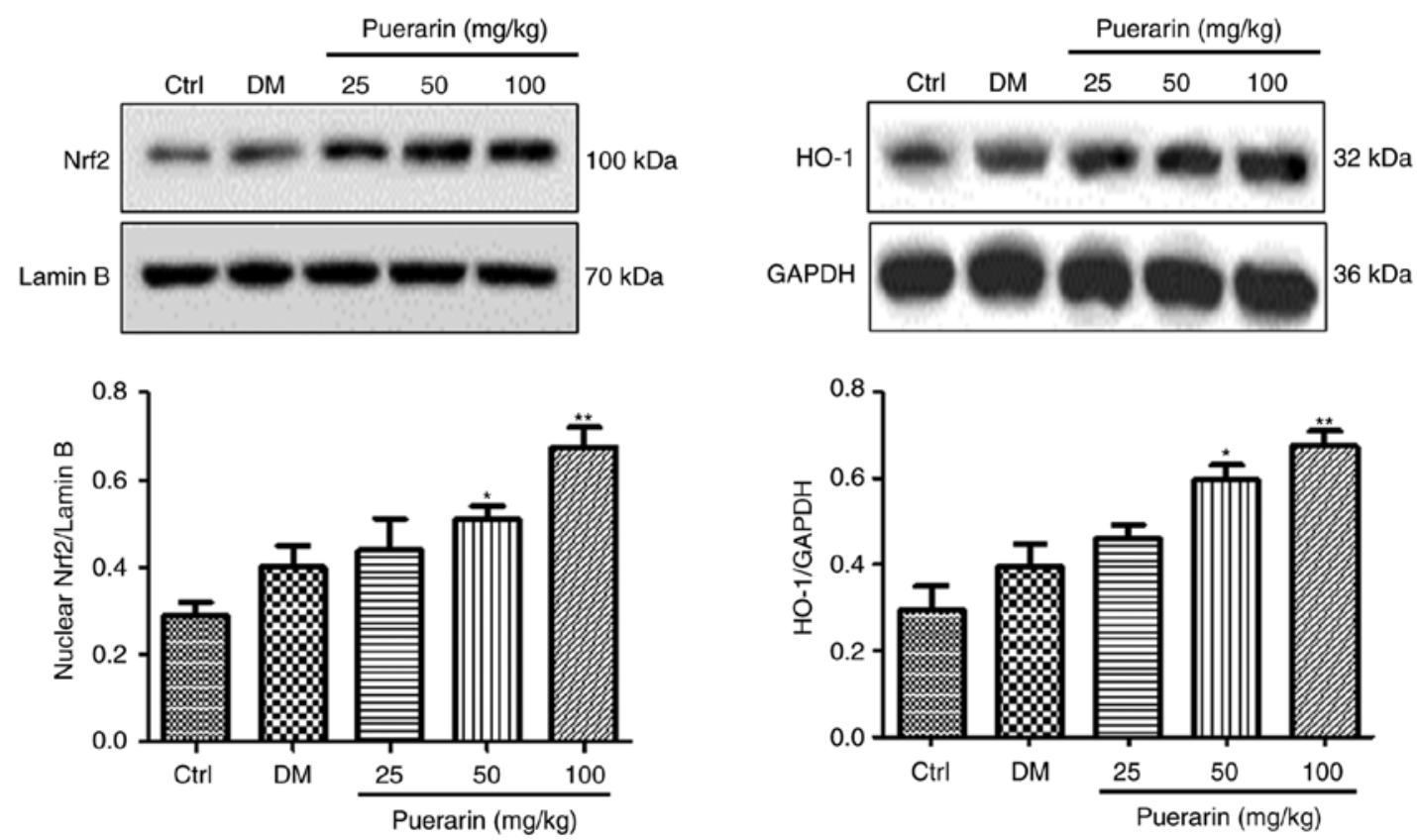

Figure 6. Puerarin administration increases the protein expression levels of members of the Nrf2/HO-1 signaling pathway in streptozocin-induced diabetic rats. Western blot analysis was used in retina tissues from the different experimental groups ( $\mathrm{n}=6$ rats/group) to quantify the protein expression levels of Nrf2 and HO-1. ${ }^{*} \mathrm{P}<0.05,{ }^{* * *} \mathrm{P}<0.01$ vs. DM. Ctrl, untreated control rats; DM, untreated diabetic rats; Nrf2, nuclear factor erythroid 2 like 2; HO-1, heme oxygenase-1.

an important antioxidant agent in the retina and serves a role similar to vitamins $\mathrm{A}, \mathrm{E}$ and $\mathrm{C}$, and selenium in the defense against cataract formation (46). In the present study, the levels of GSH in puerarin-treated rats were higher compared with untreated diabetic rats. Retinal inflammatory factors, such as IL-1 $\beta$ and VEGF, have been previously associated with cataract development (47); the present ELISA results suggested that puerarin induced a decrease in the protein expression levels of IL-1 $\beta$ and VEGF.

Cataract is a common complication of diabetes. In addition to oxidative stress, diabetic cataracts are caused by the accumulation of polyols within the lenses of patients with diabetes (48). In the lens, insulin is not involved in the regulation of the levels of glucose or other sugars (49). The glucose present at high levels in the aqueous humor can diffuse passively into the lens, where aldose reductase converts glucose into sorbitol and galactose into galactitol. These resulting polyols cannot diffuse from the lens to the aqueous humor (50). Therefore, the uptake of sodium in the aqueous humor increases, leading to swelling, electrolyte imbalance (51) and cataract formation. Puerarin is an isoflavone, and a potent inhibitor of aldose reductase (52). Therefore, in the present study, the antioxidative effects of puerarin were investigated.

Nrf2 is a member of the 'cap'n'collar' family of transcription factors, and serves an important role in protecting cells via antioxidative mechanisms (53). A previous study demonstrated that the Kelch-like ECH-associating protein 1/Nrf2/antioxidant response element (ARE) pathway is one of the most important pathways in the intracellular antioxidant and cytotoxic defense system (54). Nrf2 is an important 
transcription factor involved in the regulation of cell responses to oxidative stress $(55,56)$ and interacts with AREs to induce the expression of detoxifying enzymes, such as HO-1, a phase-II detoxifying enzyme (57). A previous study has demonstrated that HO-1 catalyzes the conversion of heme into biliverdin, carbon monoxide (CO) and ferric iron (58). In addition, biliverdin has antioxidant properties and is an effective free radical scavenger. Ferric iron facilitates the binding between ferritin and free iron, thus reducing oxidative stress (59). Therefore, ferric iron is able to attenuate oxidative toxicity (59). Endogenous $\mathrm{CO}$ is a gaseous cellular messenger molecule that is involved in many biological processes (60). In addition, HO-1 is related to reductions in lipid peroxidation and caspase- 3 activity, as well as apoptosis resistance and tumor necrosis factor- $\alpha$ inhibition (61). Nrf2/HO-1 pathway is activated when damaged and when a drug acts on the animal, the drug can activate $\mathrm{Nrf} / \mathrm{HO}-1$ signaling as a protective measure (62). Although many previous studies have reported that the Nrf2/HO-1 pathway is involved in the development of various diseases $(63,64)$, to the best of our knowledge, the present study is the first to suggest that puerarin served protective effects on cataract development partly through the Nrf2/HO-1 pathway.

Collectively, the present study demonstrated that puerarin could prevent cataract development and progression partly through the Nrf2/mHO-1 signaling pathway in the retina, exhibiting antioxidative effects in STZ-induced diabetic rats. The present findings provide insight into the antioxidative mechanisms underlying puerarin action in the treatment of cataract.

\section{Acknowledgements}

Not applicable.

\section{Funding}

No funding was received.

\section{Availability of data and materials}

The datasets used and/or analyzed during the current study are available from the corresponding author on reasonable request.

\section{Authors' contributions}

DZ and ML collaborated to the conception and design of the study. DZ and ML contributed to acquisition, analysis, and interpretation of the data in writing the manuscript. Both authors read and approved the final manuscript.

\section{Ethics approval and consent to participate}

The present study was approved by the Laboratory Animal Management Committee of Linyi Central Hospital (approval no. 2006-398).

\section{Patient consent for publication}

Not applicable.

\section{Competing interests}

The authors declare that they have no competing interests.

\section{References}

1. Sen S and Chakraborty R: Treatment and diagnosis of diabetes mellitus and its complication: Advanced approaches. Mini Rev Med Chem 15: 1132-1133, 2015.

2. Li L, Wan XH and Zhao GH: Meta-analysis of the risk of cataract in type 2 diabetes. BMC Ophthalmol 14: 94, 2014.

3. Gardiner TA, Archer DB, Curtis TM and Stitt AW: Arteriolar involvement in the microvascular lesions of diabetic retinopathy: Implications for pathogenesis. Microcirculation 14: 25-38, 2007.

4. Javadi MA and Zareighanavati S: Cataracts in diabetic patients: A review article. J Ophthalmic Vis Res 3: 52-65, 2008.

5. Murphy P, Kabir MH, Srivastava T, Mason ME, Dewi CU, Lim S, Yang A, Djordjevic D, Killingsworth MC, Ho JWK, et al: Light-focusing human micro-lenses derived from zebrafish-like lens cell masses model lens development and drug-induced cataract in vitro. Development 145: pii: dev155838, 2018. doi: $10.1242 /$ dev.155838.

6. Chua J, Koh JY, Tan AG, Zhao W, Lamoureux E, Mitchell P, Wang JJ, Wong TY and Cheng CY: Ancestry, socioeconomic status, and age-related cataract in asians: The Singapore epidemiology of eye diseases study. Ophthalmology 122: 2169-2178, 2015.

7. Head KA: Natural therapies for ocular disorders, part two: Cataracts and glaucoma. Altern Med Rev 6: 141-166, 2001.

8. Ross WM, Creighton MO and Trevithick JR: Radiation cataractogenesis induced by neutron or gamma irradiation in the rat lens is reduced by vitamin E. Scanning Microsc 4: 641-650, 1990.

9. Brown L, Rimm EB, Seddon JM, Giovannucci EL, Chasan-Taber L, Spiegelman D, Willett WC and Hankinson SE: A prospective study of carotenoid intake and risk of cataract extraction in US men. Am J Clin Nutr 70: 517-524, 1999.

10. Varma SD, Kumar S and Richards RD: Light-induced damage to ocular lens cation pump: Prevention by vitamin C. Proc Natl Acad Sci USA 76: 3504-3506, 1979.

11. Zhou YX, Zhang H and Peng C: Puerarin: A review of pharmacological effects. Phytother Res 28: 961-975, 2014.

12. Xie RQ, Du J and Hao YM: Myocardial protection and mechanism of puerarin injection on patients of coronary heart disease with ischemia/reperfusion. Zhongguo Zhong Xi Yi Jie He Za Zhi 23: 895-897, 2003 (In Chinese).

13. Jiang K, Chen H, Tang K, Guan W, Zhou H, Guo X, Chen Z, Ye Z and $\mathrm{Xu} \mathrm{H}$ : Puerarin inhibits bladder cancer cell proliferation through the mTOR/p70S6K signaling pathway. Oncol Lett 15: 167-174, 2018.

14. Lorenzen J, Kumarswamy R, Dangwal S and Thum $\mathrm{T}$ : MicroRNAs in diabetes and diabetes-associated complications. RNA Biol 9: 820-827, 2012.

15. Zhao CY, Hou LH and Che HX: Influence of puerarin eye drops for the optic disc parameters and antioxidant capacity of patients with glaucoma. Int Eye Sci 15: 1332-1334, 2015.

16. Liu CM, Ma JQ, Liu SS, Feng ZJ and Wang AM: Puerarin protects mouse liver against nickel-induced oxidative stress and inflammation associated with the TLR4/p38/CREB pathway. Chem Biol Interact 243: 29-34, 2016.

17. Ma JQ, Ding J, Xiao ZH and Liu CM: Puerarin ameliorates carbon tetrachloride-induced oxidative DNA damage and inflammation in mouse kidney through ERK/Nrf2/ARE pathway. Food Chem Toxicol 71: 264-271, 2014.

18. Jun-Hua L, Zhang SP and Cong-Rong YU: Effect of puerarin on blood pressure and serum lipid in a rat model of insulin resistance. Chin J Pathophysiol 22: 997-1000, 2006 (In Chinese).

19. Chen WC, Hayakawa S, Yamamoto T, Su HC, Liu IM and Cheng JT: Mediation of beta-endorphin by the isoflavone puerarin to lower plasma glucose in streptozotocin-induced diabetic rats. Planta Med 70: 113-116, 2004.

20. Hao LN, Ling YL, Zhen-Yong GU, Huang XL and Shou-Zhi HE: Effects of puerarin on inducible nitric oxide synthase in lens during diabetic cataract in rats. Chin J Pathophysiol 4: 620-626, 2004

21. Qu L, Chen H, Liu X, Bi L, Xiong J, Mao Z and Li Y: Protective effects of flavonoids against oxidative stress induced by simulated microgravity in SH-SY5Y cells. Neurochem Res 35: 1445-1454, 2010. 
22. Rong L, Yang XD, Chen LJ, Wang AQ and He LF: New research progresses on and mechanism pharmacological effects of puerarin. Popular Sci Technol 16: 138-142, 2014.

23. Hao LN, Wang M, Ma JL and Yang T: Puerarin decreases apoptosis of retinal pigment epithelial cells in diabetic rats by reducing peroxynitrite level and iNOS expression. Sheng Li Xue Bao 64: 199-206, 2012.

24. Hao LN, He SZ, Shen YH, Zhang YQ, Wang ZY and Wang YH: Protective effects of puerarin on lens epithelial cells in rat diabetic cataract. Zhonghua Yan Ke Za Zhi 47: 320-326, 2011 (In Chinese)

25. Teng Y, Cui H, Yang M, Song H, Zhang Q, Su Y and Zheng J: Protective effect of puerarin on diabetic retinopathy in rats. Mol Biol Rep 36: 1129-1133, 2009.

26. Yuan W, Pan QI, Chen G, Yan J, Xia J and Chen Y: E-cadherin expression in a rat model of acute pancreatitis. Exp Ther Med 10: 2088-2092, 2015

27. Dang M, Zeng X, Wang H, Li H, Du F, Chen B and Guo C: GW28-e0833 Inhibition of myocardial ischemia/reperfusion apoptosis by soluble receptor for advanced glycation end-product (sRAGE) via interferon-induced immunoproteasome activity. J Am Coll Cardiol 70: C31-C32, 2017.

28. Park JH, Lee YJ, Kim JJ, Shin YC and Kim JC: The effect of pinitol on cataractogenesis and anti-oxidative effect in streptozotocin induced diabetic rats. J Korean Ophthalmol Soc 46: 1886-1893, 2005

29. Xiaojian G, Qiuyan Z and Shuhua T: Inhibitory effect of r-hirudin variant III on streptozotocin-induced diabetic cataracts in rats. Scientific World J 2013: 630651, 2013.

30. Arnal E, Miranda M, Almansa I, Muriach M, Barcia JM, Romero FJ, Diaz-Llopis M and Bosch-Morell F: Lutein prevents cataract development and progression in diabetic rats. Graefes Arch Clin Exp Ophthalmol 247: 115-120, 2009.

31. Yao XJ, Yin JA, Xia YF, Xia YF, Wei ZF, Luo YB, Liu M, Feleder C and Dai Y: Puerarin exerts antipyretic effect on lipopolysaccharide-induced fever in rats involving inhibition of pyrogen production from macrophages. J Ethnopharmacol 141: 322-330, 2012.

32. Zhao SS, Yang WN, Jin H, Ma KG and Feng GF: Puerarin attenuates learning and memory impairments and inhibits oxidative stress in STZ-induced SAD mice. Neurotoxicology 51: 166-171, 2015.

33. Varma SD: Scientific basis for medical therapy of cataracts by antioxidants. Am J Clin Nutr 53 (Suppl 1): S335-S345, 1991.

34. Kowluru RA and Kanwar M: Effects of curcumin on retinal oxidative stress and inflammation in diabetes. Nutr Metab (Lond) 4: 8, 2007.

35. Zelinová V, Mistrík I, Pavlovkin J and Tamás L: Glutathione peroxidase expression and activity in barley root tip after short-term treatment with cadmium, hydrogen peroxide and t-butyl hydroperoxide. Protoplasma 250: 1057-1065, 2013.

36. Livak KJ and Schmittgen TD: Analysis of relative gene expression data using real-time quantitative PCR and the 2(-Delta Delta C(T)) method. Methods 25: 402-408, 2001

37. Sun W, Zheng XZ, Xu QL, Nian H and Liu GL: Effects of puerarin on ADRP gene expression in fatty tissue of type 2 diabetes mellitus rats. Zhongguo Zhong Yao Za Zhi 33: 2026-2028, 2008 (In Chinese).

38. Hao LN, Ling YQ, Luo XM, Mao YX, Mao QY, He SZ and Ling YL: Puerarin decreases lens epithelium cell apoptosis induced partly by peroxynitrite in diabetic rats. Sheng Li Xue Bao 58: 584-592, 2006.

39. Wan L, Liu WB, Shen YY, Yu QL and Zhang JJ: Oxidative stress-apoptosis mediated STZ-induced diabetic cataract and the interventions of puerarin. Int J Ophthalmol 14: 1773-1775, 2014.

40. Xu X, Zheng N, Chen Z, Huang W, Liang T and Kuang H: Puerarin, isolated from Pueraria lobata (Willd.), protects against diabetic nephropathy by attenuating oxidative stress. Gene 591: 411-416, 2016.

41. Cai Y, Zhang X, Xu X and Yu Y: Effects of puerarin on the retina and STAT3 expression in diabetic rats. Exp Ther Med 14: 5480-5484, 2017

42. Babizhayev MA, Vishnyakova KS and Yegorov YE: Telomeredependent senescent phenotype of lens epithelial cells as a biological marker of aging and cataractogenesis: The role of oxidative stress intensity and specific mechanism of phospholipid hydroperoxide toxicity in lens and aqueous. Fundam Clin Pharmacol 25: 139-162, 2011.

43. Katta AV, Katkam RV and Geetha H: Lipid peroxidation and the total antioxidant status in the pathogenesis of age related and diabetic cataracts: A study on the lens and blood. J Clin Diagn Res 7: 978-981, 2013.

44. Li S, Yue J, Zhou W and Li L: An investigation into the preparation, characterization and antioxidant activity of puerarin/cyclodextrin inclusion complexes. J Inclusion Phenomena Macrocyclic Chem 82: $1-8,2015$.
45. Cui X, Gong J, Han H, He L, Teng Y, Tetley T, Sinharay R, Chung KF, Islam T, Gilliland $\mathrm{F}$, et al: Relationship between free and total malondialdehyde, a well-established marker of oxidative stress, in various types of human biospecimens. J Thorac Dis 10: 3088-3097, 2018

46. Kamegawa M, Nakanishi-Ueda T, Iwai S, Ueda T, Kosuge S, Ogura H, Sasuga K, Inagaki M, Watanabe M, Oguchi K, et al: Effect of lipid-hydroperoxide-induced oxidative stress on vitamin $\mathrm{E}$, ascorbate and glutathione in the rabbit retina. Ophthalmic Res 39: 49-54, 2007.

47. Gologorsky D, Thanos A and Vavvas D: Therapeutic interventions against inflammatory and angiogenic mediators in proliferative diabetic retinopathy. Mediators Inflamm 2012: 629452, 2012.

48. Abdul Nasir NA, Agarwal R, Sheikh Abdul Kadir SH, Vasudevan S, Tripathy M, Iezhitsa I, Mohammad Daher A, Ibrahim MI and Mohd Ismail N: Reduction of oxidative-nitrosative stress underlies anticataract effect of topically applied tocotrienol in streptozotocin-induced diabetic rats. PLoS One 12: e0174542, 2017.

49. Levari R, Wertheimer E, Berman ER and Kornblueth W: Effect of insulin on pathways of glucose oxidation in the rat lens. Nature 192: 1075-1076, 1961.

50. Sabah JR, Davidson H, Mcconkey EN and Takemoto L: In vivo passage of albumin from the aqueous humor into the lens. Mol Vis 10: 254-259, 2004.

51. Drozdov AD and deClaville Christiansen J: Modeling the effects of $\mathrm{pH}$ and ionic strength on swelling of anionic polyelectrolyte gels. J Chem Phys 142: 114904, 2015.

52. Feng CG, Zhang LX and Liu X: Progress in research of aldose reductase inhibitors in traditional medicinal herbs. Zhongguo Zhong Yao Za Zhi 30: 1496-1500, 2005 (In Chinese).

53. Kobayashi A, Ohta T and Yamamoto M: Unique function of the Nrf2-Keapl pathway in the inducible expression of antioxidant and detoxifying enzymes. Methods Enzymol 378: 273-286, 2004.

54. Magesh S, Chen Y and Hu L: Small molecule modulators of Keap1-Nrf2-ARE pathway as potential preventive and therapeutic agents. Med Res Rev 32: 687-726, 2012

55. Nakagami Y: Nrf2 is an attractive therapeutic target for retinal diseases. Oxid Med Cell Longev 2016: 7469326, 2016.

56. Foresti R, Bucolo C, Platania CM, Drago F, Dubois-Randé JL and Motterlini R: Nrf2 activators modulate oxidative stress responses and bioenergetic profiles of human retinal epithelial cells cultured in normal or high glucose conditions. Pharmacol Res 99: 296-307, 2015.

57. Chen CY, Jang JH, Li MH and Surh YJ: Resveratrol upregulates heme oxygenase-1 expression via activation of NF-E2-related factor 2 in PC12 cells. Biochem Biophys Res Commun 331: 993-1000, 2005.

58. Kim KC, Kang KA, Rui Z, Piao MJ, Kim GY, Kang MY, Lee SJ, Lee NH, Surh YJ and Hyun JW: Up-regulation of Nrf2-mediated heme oxygenase-1 expression by eckol, a phlorotannin compound, through activation of Erk and PI3K/Akt. Int J Biochem Cell Biol 42: 297-305, 2010.

59. Finazzi D and Arosio P: Biology of ferritin in mammals: An update on iron storage, oxidative damage and neurodegeneration. Arch Toxicol 88: 1787-1802, 2014

60. Bucolo C and Drago F: Carbon monoxide and the eye: Implications for glaucoma therapy. Pharmacol Ther 130: 191-201, 2011.

61. Zhou W, Yuan X, Zhang L, Su B, Tian D, Li Y, Zhao J, Wang Y and Peng S: Overexpression of HO-1 assisted PM2.5-induced apoptosis failure and autophagy-related cell necrosis. Ecotoxicol Environ Saf 145: 605-614, 2017.

62. Lou J, Cao G, Li R, Liu J, Dong Z and Xu L: $\beta$-caryophyllene attenuates focal cerebral ischemia-reperfusion injury by Nrf2/HO-1 pathway in rats. Neurochem Res 41: 1291-1304, 2016.

63. He M, Pan H, Chang RC, So KF, Brecha NC and Pu M: Activation of the Nrf2/HO-1 antioxidant pathway contributes to the protective effects of Lycium barbarum polysaccharides in the rodent retina after ischemia-reperfusion-induced damage. PLoS One 9: e84800, 2014

64. Yalniz M, Demirel U, Orhan C, Bahcecioglu IH, Ozercan IH, Aygun C, Tuzcu M and Sahin K: Nadroparin sodium activates Nrf2/HO-1 pathway in acetic acid-induced colitis in rats. Inflammation 35: 1213-1221, 2012.

This work is licensed under a Creative Commons Attribution-NonCommercial-NoDerivatives 4.0 International (CC BY-NC-ND 4.0) License. 